Supporting information:

\title{
Quantitative Differentiation of Cell Surface-Bound and Internalized Cationic Gold Nanoparticles Using Mass Spectrometry
}

Singyuk Hou, $\uparrow$ Kristen N. Sikora, $\uparrow$ Rui Tang, Yuanchang Liu, Yi-Wei Lee, Sung Tae

Kim, Ziwen Jiang, Richard W. Vachet* and Vincent M. Rotello*

$\dagger$ S. Hou and K. N. Sikora contributed equally to this work.

Department of Chemistry, University of Massachusetts Amherst, 710 North Pleasant Street, Amherst, Massachusetts, 01003, USA

*rwvachet@chem.umass.edu (R.W.V.), rotello@chem.umass.edu (V.M.R.) 


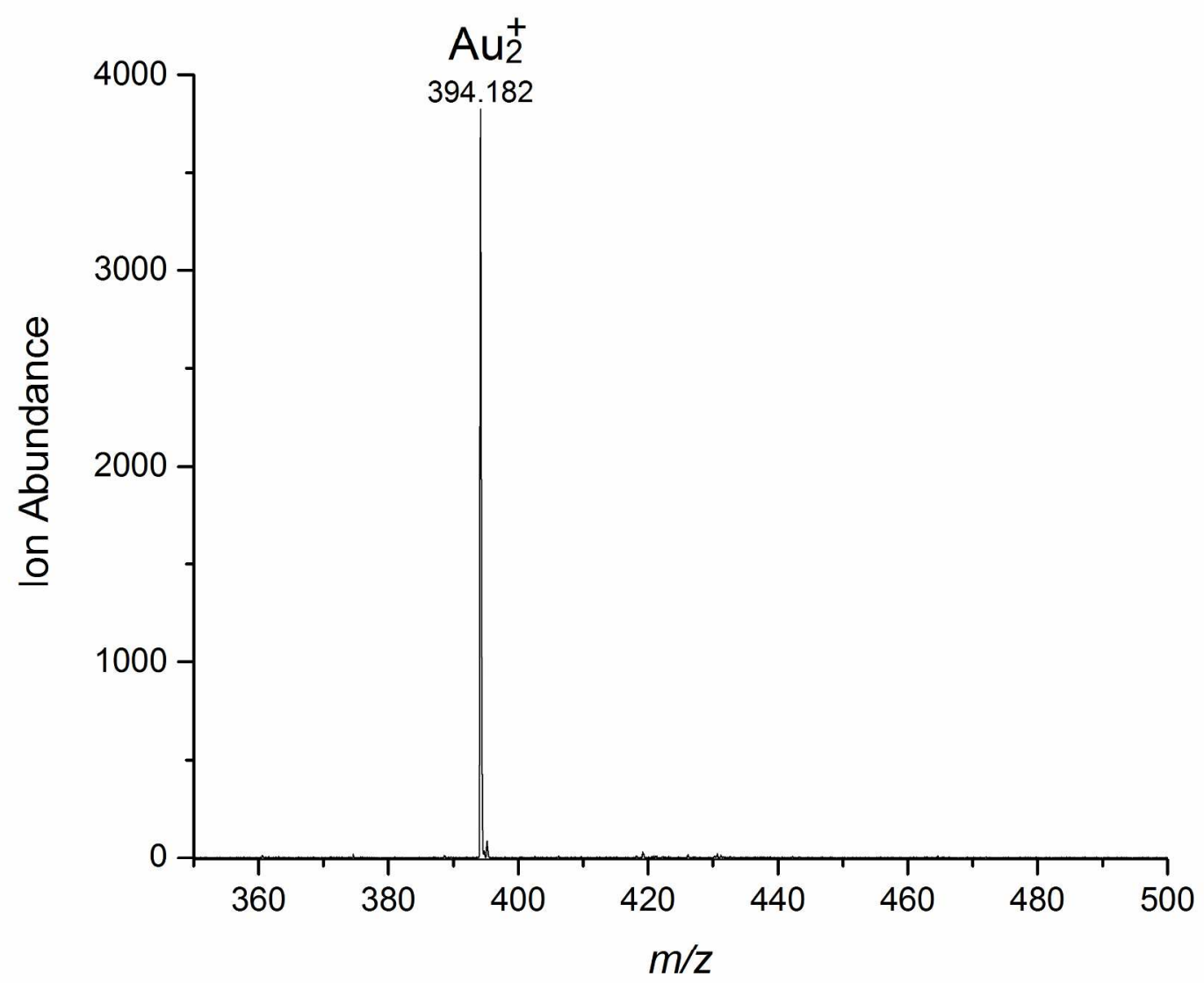

Figure S1. LDI-MS detection of AuNPs on plain slide after washing steps, 250 nM AuNP 1 media solution was incubated on poly-lysine coated glass slide for 30 minutes at $37^{\circ} \mathrm{C}$. After the incubation, the AuNP 1 solution was removed and the glass slide was washed with PBS for 5 times, AuNP 2 was then incubated for 30 minutes under the same condition and washed with PBS for one time. 


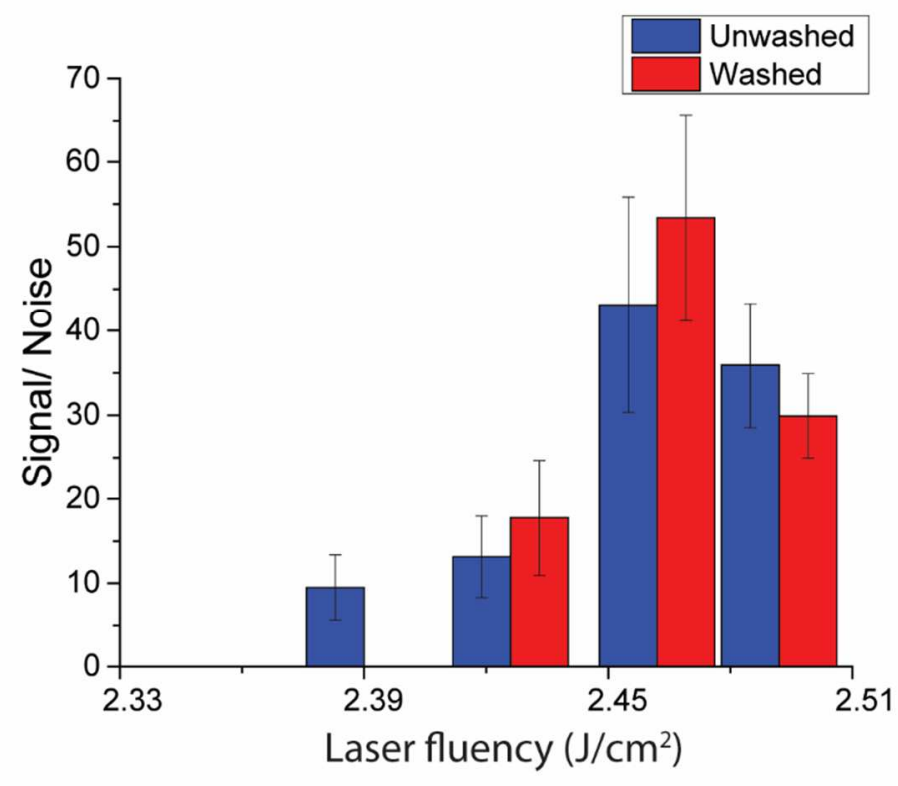

Figure S2. LDI-MS of AuNP 2 on cell monolayers before and after washing. 250 nM AuNP 2 was incubated with the cell monolayer for 60 minutes in serum free media at $37^{\circ} \mathrm{C}$. After incubation, the cell monolayer was either washed four times (washed) or one time (unwashed) before LDI-MS analysis. 

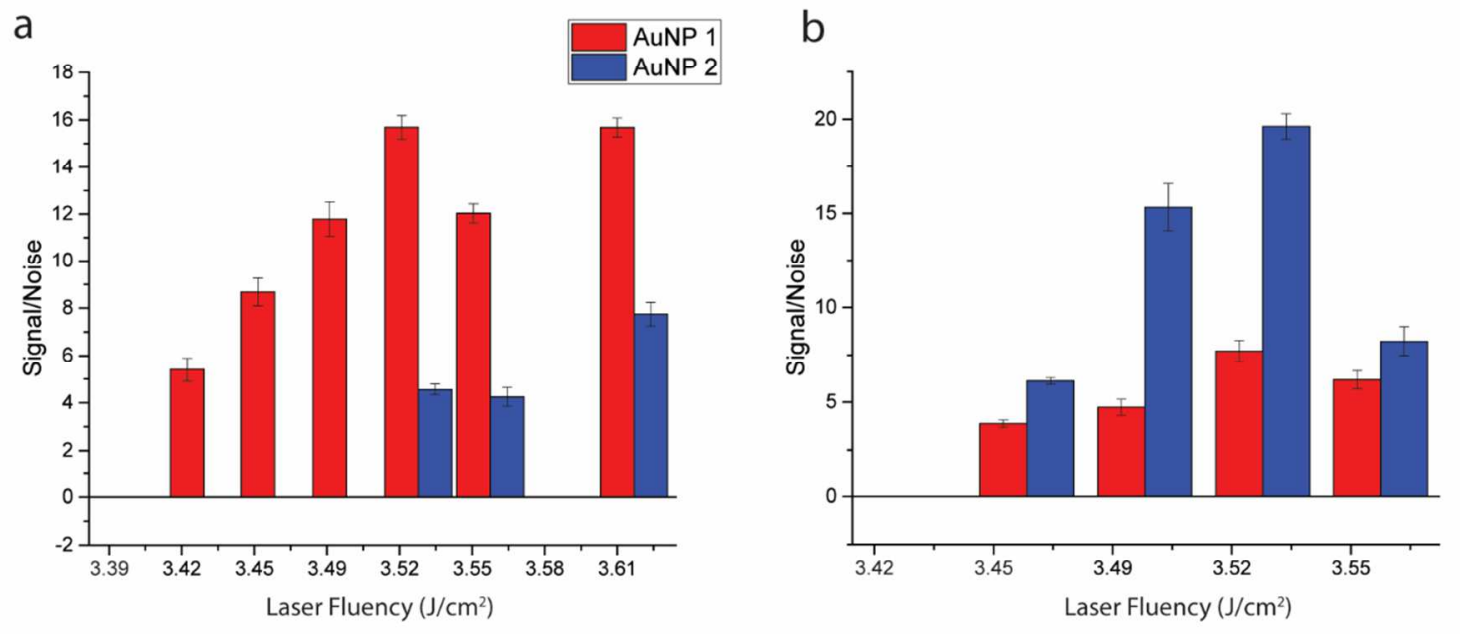

Figure S3. a) LDI-MS of HeLa cell monolayer after sequential incubation by AuNP 2 and AuNP 1. b) LDIMS of HeLa cells monolayers treated with $3 \mathrm{mg} / \mathrm{ml} \mathrm{NaN}_{3}$ and $50 \mathrm{nM}$ 2-deoxyglucose in DMEM for 30 minute prior to sequential AuNP incubation. 
a
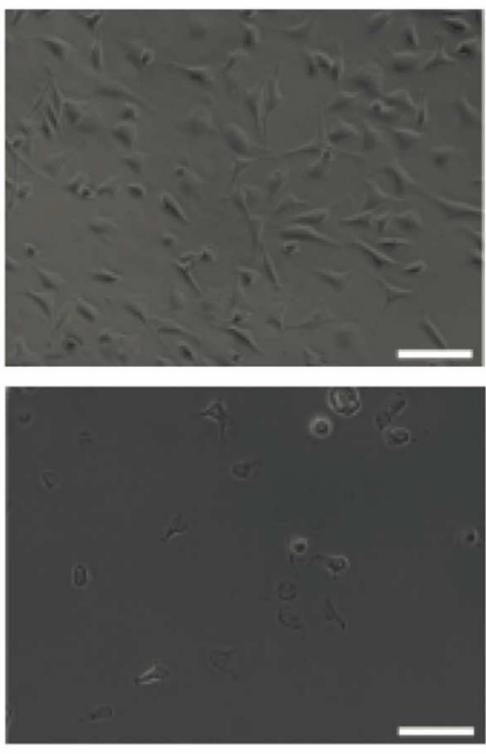

b

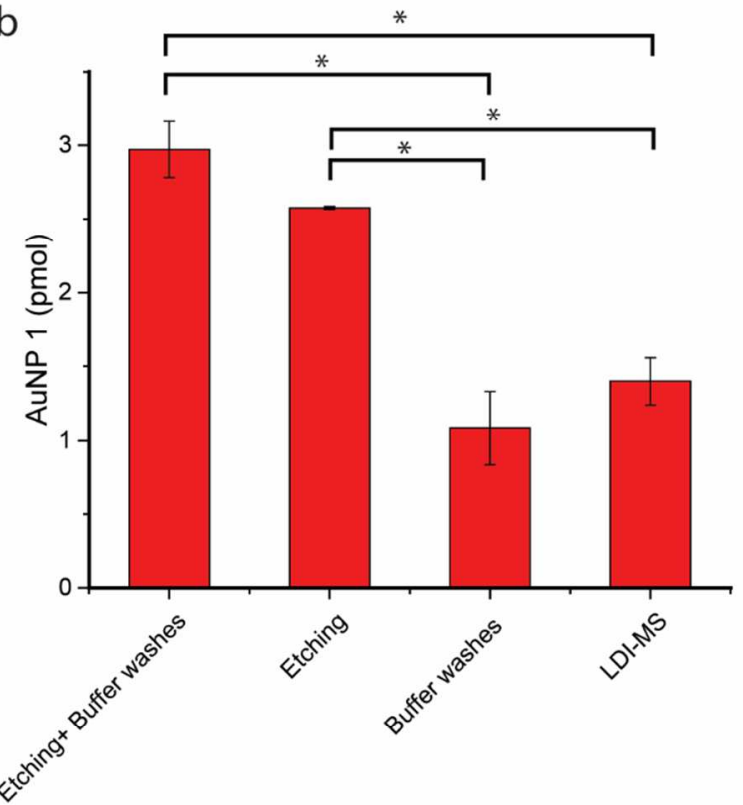

Figure S4. a) Microscopic image of HeLa cells not etched (up) and etched (down) by $\mathrm{I}_{2} / \mathrm{KI}$ solution. Scale bars: $100 \mu \mathrm{m}$. b) Comparisons of removal of surface-bound AuNPs via etching and buffer washing measured by ICP-MS. LDI-MS measurement of cell-surface adhered AuNP was added for further comparison. All the experiments were done with HeLa cells incubated with AuNP 1 for 30 minutes. The gold amount measured by ICP-MS in the etched and non-etched samples were converted to AuNP amount through division by $48.62 \mathrm{ng}$ gold/ pmol AuNP. One way-ANOVA $(\mathrm{P}<0.05)$ was performed, $\mathrm{n}=3$, all error bars represent standard deviation. Stars above the bars indicate significance, whereas no stars suggest not significantly different. 

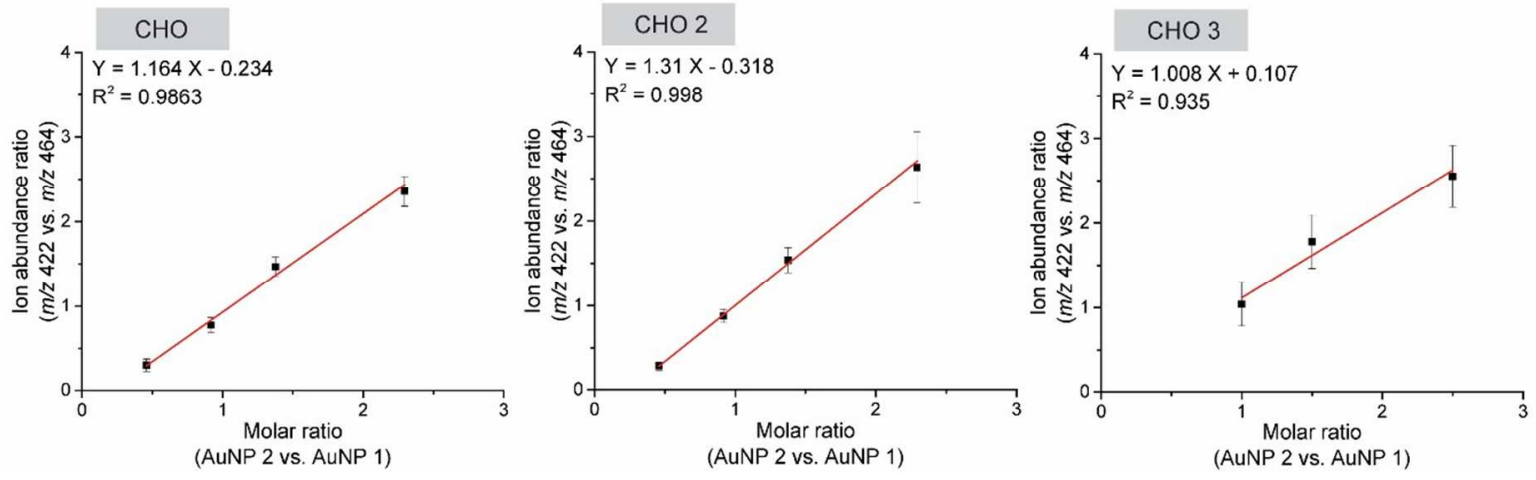

Figure S5. LDI-MS calibration curves for AuNP 2 on the cell surface. HeLa, CHO, CHO 2 and CHO 3 cells were cultured on ITO-glass slide. Increasing concentrations of AuNP 2 were mixed with AuNP 1 (internal standard) and incubated with cells as described in the text. Cells on the glass slide were detected using $\mathbf{3 8 . 6}$ $\mu \mathrm{J}$ to only detect AuNPs on cell surface. Molecular ions of both AuNPs were plotted against molar ratios. 

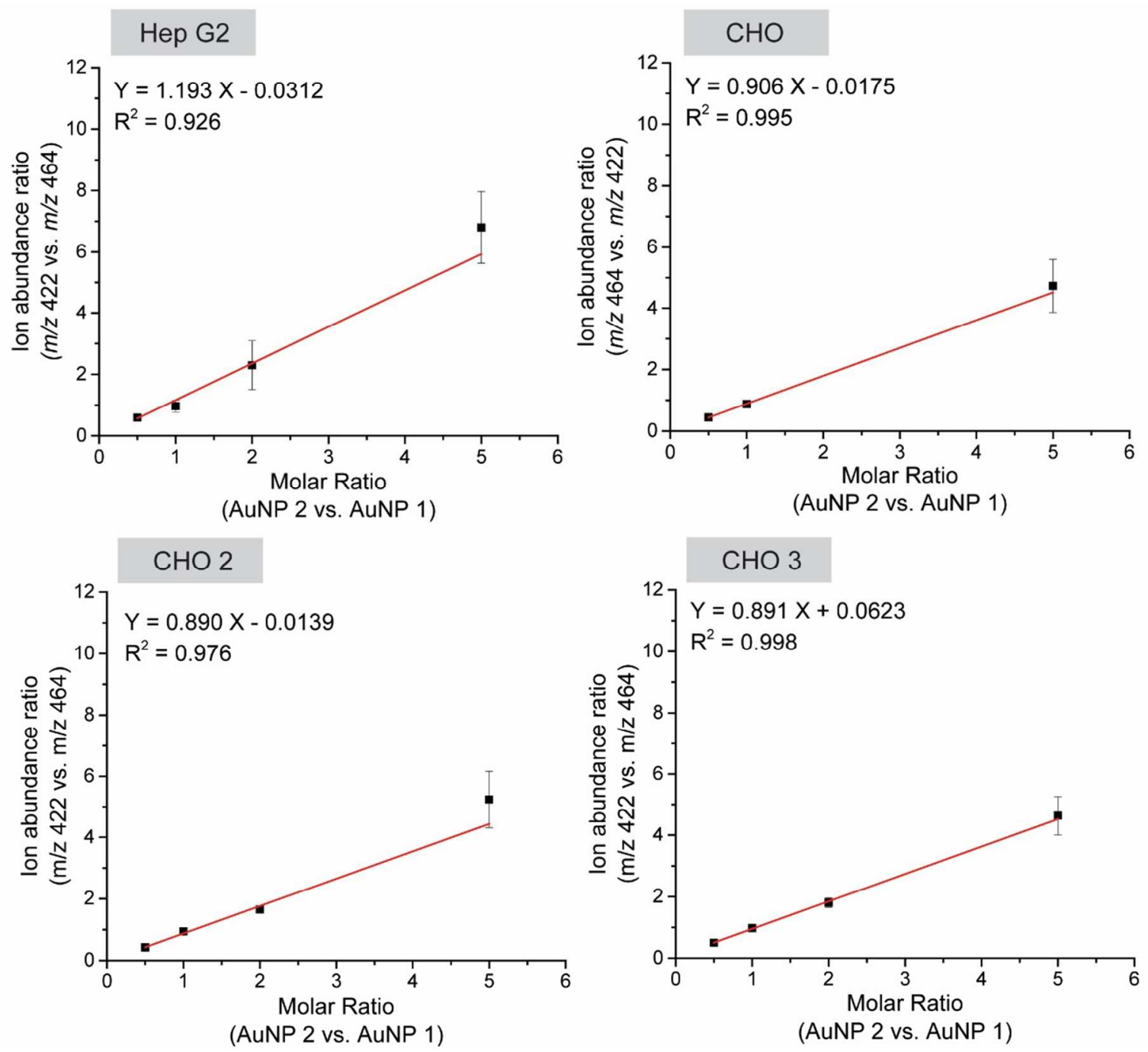

Figure S6. LDI-MS calibration curves for AuNP 2 in cell lysate. Increasing concentrations of AuNP 2 were mixed with AuNP 1 (internal standard) and spiked into cell lysate of HeLa, Hep G2, CHO, CHO 2 and CHO 3 cells. After centrifugation, the resulting pellets were deposited on a stainless steel target and analyzed with LDI-MS. Molecular ions of both AuNPs were plotted against molar ratios. 

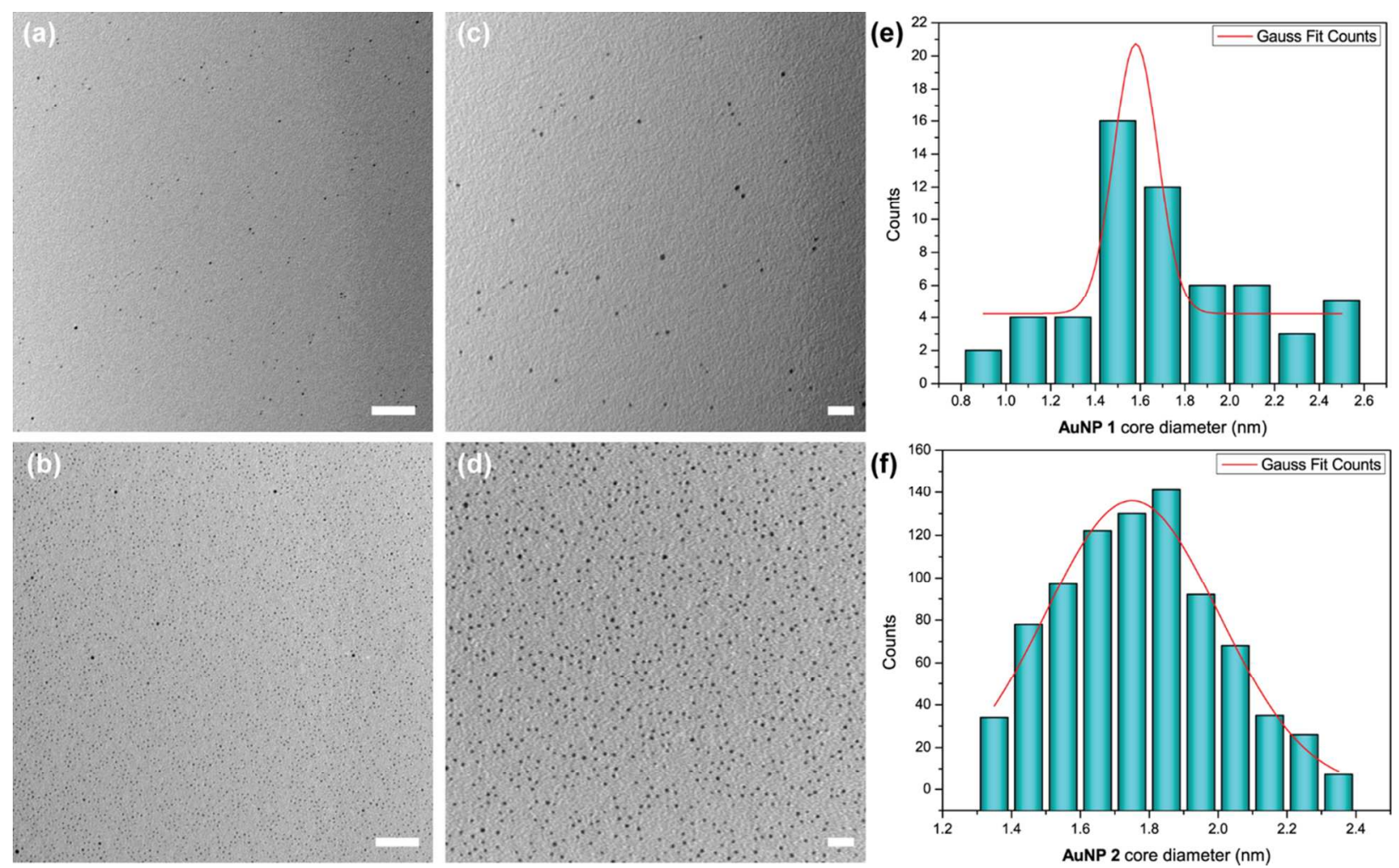

Figure S7. (a) (d) TEM images of AuNPs: (a) \& (c) AuNP 1; (b) \& (d) AuNP 2. The white scale bar is $50 \mathrm{~nm}$ in (a) \& (d) and $10 \mathrm{~nm}$ in (c) \& (d). (e), (f) Core size distribution analysis for AuNPs corresponding to (c), (d), respectively: (e) AuNP 1: (1.6 \pm 0.1$) \mathrm{nm}$, and (f) AuNP 2: (1.7 \pm 0.2$) \mathrm{nm}$.
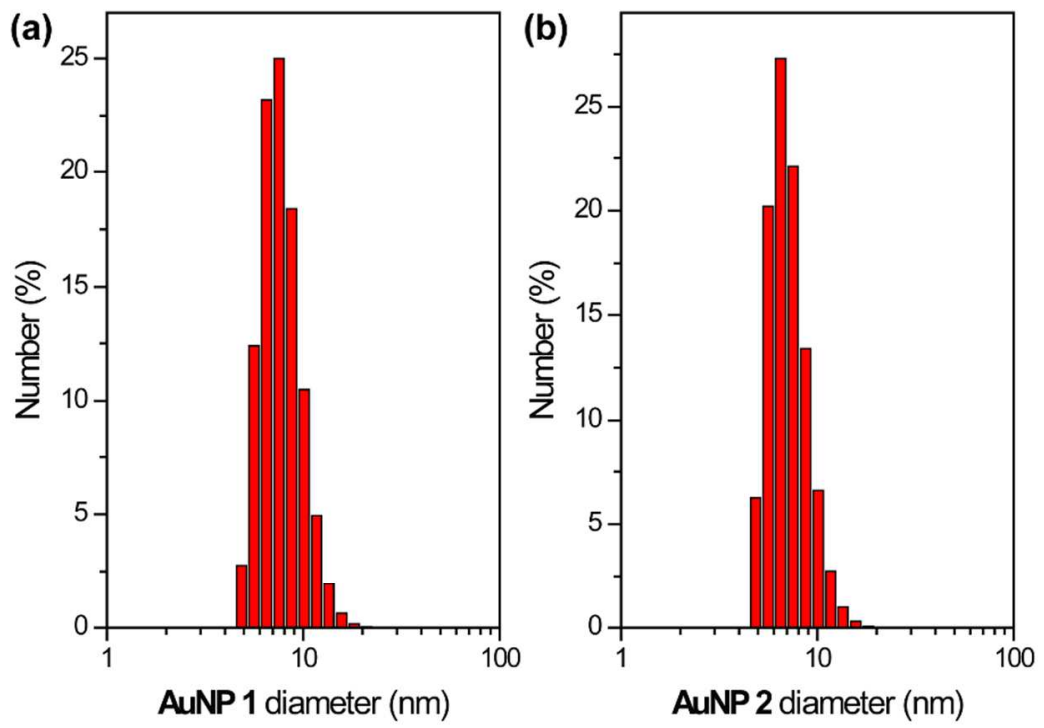

Figure S8. DLS histogram of AuNPs, demonstrating the hydrodynamic diameter distribution of nanoparticles:

(a) AuNP

and

(b)

AuNP

2. 
Calculation of absolute quantity of total, internalized and cell surface-bound AuNP 2 by coupling LDI-MS and ICP-MS

After incubation and addition of the internal standard (AuNP 1), the cells are lysed for ICP-MS detection. The gold amount measured from the sample is denoted by $\mathrm{X}$ (ng). $\mathrm{X}$ arises from contributions from the gold amounts of AuNP 2 (X (AuNP 2) total) and AuNP 1 (X (AuNP 1)). The LDI-MS measured molar ratio between AuNP 2 and AuNP 1 is denoted by Y. Ytotal represents the molar ratio of the total amounts of AuNP 2 to AuNP 1. With equation (1), the absolute amount of total AuNP 2 with cells can be calculated.

Equation (2) describes the absolute amount of AuNP 2 on the cell surface. Ysurface represents the molar ratio of cell surface-bound AuNP 2 to AuNP 1. By subtracting AuNP 2 on cell surface from total amount of AuNP 2 in equation (3), absolute amount for internalization can be determined.

$$
\begin{aligned}
& \left\{\begin{array}{c}
X(\text { AuNP 2 })_{\text {total }}=X-X(\text { AuNP 1) } \\
\frac{X(\text { AuNP 2 })_{\text {total }}}{X(\text { AuNP 1 })}=Y_{\text {total }}
\end{array}\right. \\
& X(A u N P 2)_{\text {surface }}=\frac{Y_{\text {surface }}}{Y_{\text {total }}} \times X(A u N P 2)_{\text {total }} \\
& X(A u N P 2)_{\text {internalization }}=X(A u N P 2)_{\text {total }}-X(A u N P 2)_{\text {surface }}
\end{aligned}
$$

